\title{
The Emotional Labor in the Interaction of Mobile Game Live Streaming on Douyin
}

\author{
XiaoHan Yang ${ }^{1, a}$ \\ ${ }^{1}$ Room 1008, Party and Government Comprehensive Building, Xiangcheng Administrative New District, Henan \\ Province \\ ${ }^{a}$ Email: xiaohany3@student.unimelb.edu.au
}

\begin{abstract}
The live streaming function of Douyin, a social application, has occupied a great deal of time in the lives of many young Chinese people. Based on the theory of the magic circle, remediation, narratology and fan economy, this research finds that there is emotional labor in the interaction between live streamers and audiences in the live streaming of mobile games on Douyin. Moreover, it demonstrates that emotional labor has also constructed a new social relationship in different social circles. One is the teammate relationship in the social circle between the audience and the live streamer, and the other is the fans' class-based relationship in the social circle of audiences.
\end{abstract}

Keywords: Douyin, mobile game live streaming, emotional labor, social interaction.

\section{INTRODUCTION}

The following is a one-day work schedule of the Douyin mobile game live streamer Xiaotiantian: Get up, open Douyin, click "start live streaming," select live stream type as "game live streaming," open the mobile game Game for Peace, set up the screen, start the live streaming at 7 a.m., end at 5 p.m., and, after an hour of rest, the live streaming continues from 6 p.m. to 10 p.m. Live streaming mobile games has dominated his life, and this is not uncommon among live streamers of mobile games on Douyin.

Douyin offers various kinds of mobile game live streaming. People can enjoy the game together in the live streaming room, sharing the joy of victory and the agony of defeat. Why is game live streaming so popular? Bogost argues that the interactive nature of video games makes them an inherently compelling medium[1]. Moreover, live streaming appeals to young people because it gives them a chance to be a creator and a presenter and to be seen and heard by audiences. More importantly, live streamers can also receive financial rewards, which is an enticing prospect for young people. In any Douyin room for game live streaming, it is common to hear the live streamer say something like, "If you think I did an excellent job, please give me gifts to encourage me." There are special effects and system announcements when a member of the audience gives the live streamer a gift in the live streaming room. For example, if an audience member gives a gift called "love you," a large pink heart appears on the phone interface. Viewing live streams allows young people to connect with their favorite live streamers and with people who have similar interests and views or who are going through similar experiences. They can share comments with other members of the audience and even interact directly with live streamers. Live streaming can help young people feel less socially isolated.

The interactivity and sociality of game live streaming can offer young people a sense of carnival. Bakhtin proposed the idea of "the carnival sense of the world.[2]" He believes that the carnival allows familiar and free interaction among people. They often bring the most unlikely people together and encourage them to interact and express themselves freely in solidarity. As a carnival of young Chinese people, the live streaming function of Douyin is the banquet hall of the audience. Live streamers go there to show their game skills, and the audience goes there to use bullet screens and send gifts to interact and express themselves. Mobile game live streaming on Douyin is full of this kind of free interaction. 


\section{MAGIC CIRCLE INTERACTION: THE INTERFACE INTERACTION LAYERS IN DOUYIN'S MOBILE GAME LIVE STREAMING}

In the carnival of mobile game live streaming on Douyin, live streamers treat playing mobile games as a kind of performance. Audiences revel in the bullet screens and gift-giving. This exaggerated style of live streaming is unique to Douyin. Interaction in Douyin's mobile game live streaming can be divided into two layers: the inner layer and the outer layer.

\subsection{Inner layer-The Interactive World on the Screen}

The Dutch historian Johan Huizinga introduced the concept of the magic circle in Homo Ludens. He defined the magic circle in the following terms:

"All play moves and has its being within a playground marked off beforehand either materially or ideally, deliberately or as a matter of course. The arena, the card table, the magic circle, the temple, the stage, the screen, the tennis court, the court of justice, etc., are all in form and function playgrounds, i.e., forbidden spots, isolated, hedged round, hallowed, within which special rules obtain. All are temporary worlds within the ordinary world, dedicated to the performance of an act apart. [3]",

However, Huizinga's magic circle is not a new synonym for "game." Rather, he conceived the term to describe all places of play. The magic circle that Huizinga envisioned, like many other cultural activities, has game-like features, and so the concept of the magic circle can also be applied to video games. Playing a game means "entering a magic circle or creating one at the beginning of the game" [4].

When the live streamer enters the game, they are already in the magic circle. As an independent world, the magic circle also has specific rules, usually called game mechanics. The game mechanics determine how the game works for the players, includes the physical existence, interaction relationship, and content setting in the game. Different games have different game mechanics, just as different countries have different laws. When live streamers interact with the magic circle, the game mechanics that govern their characters depend on which game they are playing.

Conway (2010) applied the concept of the fourth wall to the magic circle[5]. The fourth wall refers to an invisible, imaginary wall that separates the actors from the audience [6]. In a game, there is an imaginary wall that separates the game character from the player. Conway (2010) believes that the game's magic circle includes the game and how players view and interact with it. Breaking the fourth wall means eliminating the wall between players and games[5]. It is better understood as repositioning the game world to include players, thereby enhancing the player's immersion in the game. The live streamer's gameplay can therefore be regarded as a new magic circle that has been expanded to include players.

There are two internal interfaces in Douyin's mobile game live streaming. When the live streamer joins the magic circle through the game character, the internal interface is the mobile game. Moreover, the audience watches the live streamer playing the game through live streaming, and from the audience's perspective, the live streamer is playing a mobile game in the internal interface. The reason for the two internal interfaces is remediation. For Bolter and Grusin, remediation is the way in which one medium is seen by society as reforming or improving upon another; it is the formal logic by which new media refashion prior media forms[7]. Audiences watching live streamers play games can be seen as reshaping the medium of games in the form of live streaming.

\subsection{Outer Layer-Interaction that Breaks Boundaries}

The outer layer is the inner interface from the audience's perspective. The magic circle shows that there are rules in the game, and behind the rules, there are hidden or visible narratives. Juul points out that games contain narrative elements. In many cases, players must play to see a specific scene or realize a particular narrative sequence[8]. The interaction and connection between the inner and outer layers are based on the magic circle narrative. Here, the magic circle narrative refers to the narrative about the content of the mobile game, including the rules of the mobile game and the live streamer's game strategy.

There are three kinds of interaction in the outer layer:

\subsubsection{The Interaction Between Audiences and Live Streamers}

The prerequisite for the audience to choose a mobile game live streaming is that they know the content of the magic circle; that is, the audience knows the game and its basic rules. In the internal interface of live streamers, the live streamer, as the main narrator, realizes the narrative through playing games. The live streamer's game character guides the development of the story of the magic circle. According to Chatman, there are three types of narrators: the absent narrator, hidden narrator, and public narrator. Based on these three narrators, narratives can also be divided into absent narratives, hidden narratives, and public narratives[9]. In an absent narrative, the reader can hardly perceive the existence of the narrative voice. In a hidden narrative, the narrator is able to express their thoughts, words and deeds in an indirect way. In a public narrative, the audience can hear 
a clear narrative voice in the text. In Douyin's mobile game live streaming, live streamers are narrators: they hide themselves in the game characters and use the characters to narrate. The audience members are readers: they usually do not notice that live streamers are narrating.

The live streamer interacts with the audience by conveying the story development in the mobile games. For example, the game characters in the mobile game Game for Peace need to land in a fixed location. During the landing, the live streamer explains where and why they chose to land. The audience then understands what the live streamer is doing and why. That is to say, the audience understands the inner narrative logic only through the live streamer's public narrative. In this case, the live streamer is a public narrator, because the audience can hear a clear narrative voice.

\subsubsection{The Interaction Between Audiences and Mobile Games}

According to Hartmann et al., when media users perceive media content as real things, media users will feel that they are in the context of space and this media environment[10]. This feeling is spatial presence. In other words, when the audience starts to feel that they are in the world created by the game, the game (or any other medium, such as books or movies) creates a sense of spatial presence for the player. When the content of the live streamer's narrative conveys a sense of immersion, the audience will feel that they are playing the mobile game instead of the live streamer. Here, the mobile game is regarded as a magic circle, so the interaction between the audience and the game can be called an imaginary magic circle interaction.

\subsubsection{The Interaction Between Audience Members}

The interaction between members of the audience is mainly carried out by bullet screens in Douyin's mobile game live streaming. The basis of this interaction is the live streamer's narrative. There may be various topics discussed in the interactions, such as the live streamer's choice of game character, the formulation of the game route, and the game equipment. Moreover, audiences also discuss their daily lives in Douyin's mobile game live streaming.

Whether it is the magic circle interaction between live streamers and mobile games in the inner layer or internal interface, or the three interactions in the outer layer, these interactions are inseparable from the mobile game itself. The mobile game is the original magic circle. After breaking the fourth wall, the original magic circle is extended. The new magic circle includes live streamers (players). Live streamers play the mobile game in this new magic circle and regards the process of playing mobile game as the live content. In the live streaming, the live streamer attracts the audience to participate in the interaction through the narrative. In this way, the magic circle is expanded once again to include the audience. Thus, the magic circle is expanded through various interactions of the mobile game live streaming. Interaction in Douyin's mobile game live streaming has an exaggerated carnival sense because live streamers and audiences use their emotions to create a good live streaming experience.

\section{THE LABOR OF THE MAGIC CIRCLE: THE EMOTIONAL LABOR OF DOUYIN MOBILE GAME LIVE STREAMING}

The concept of emotional labor emerged from indepth research on emotions. About emotion, the first thing that was noticed was emotion management. The concept of emotion management can be traced back to Aristotle. Aristotle proposed that the problem is not with emotionality, but with the appropriateness of the emotion and its expression[11]. Awareness of emotional management in Chinese culture can be traced back to the Spring and Autumn period and the Warring States period. In the Analects, Confucius says that grief should be restrained.

According to Hochschild, emotion management is also called emotional work. Emotion work has use value and occurs in situations in which people choose to regulate their emotions for their own non-compensated benefit[12]. All activities that involve interpersonal interaction involve emotions. Emotional management is required in interpersonal interactions to ensure the normal working of interpersonal relationships. Cook and Berger defined emotional work as the management of one's feelings or the work done to maintain a relationship[13]. In contrast to emotional work, which is carried out in private space and only of use value, emotional labor is emotional work completed in a paid work environment, which embodies emotional exchange value[14][15].

Hochschild stated that emotional labor is the process of managing emotions and expressing oneself to meet the emotional needs of work[16]. More specifically, employees should regulate their emotions in their interactions with customers, colleagues, and managers. This regulation of emotions includes analysis and decision-making of emotional expression, whether it is truly felt or not. The purpose of emotional labor is to create a certain feeling to satisfy customers, leading to success of the company or organization.

Emotional labor has exchange value because "it is traded and executed for wages" [15]. Emotional labor is therefore a process of commercializing human emotions. The analysis above of the interaction of Douyin's mobile game live streaming has already shown that, in live streaming, emotion is an important factor that prompts 
people to interact[17]. The ultimate goal for live streamers is to obtain more remuneration. The use of the exchange value of emotion by live streamers and audiences can therefore be regarded as emotional labor.

Emotional labor is divided into two parts: surface acting and deep acting. Surface acting occurs when employees express the emotions required for work without changing their actual feelings[16]. It can essentially be understood as employees suppressing their true but inappropriate feelings and showing feigned emotions[18]. Deep acting is a process of hard work. Through suppressing emotions, employees change their inner feelings to meet the expectations of bosses or customers, thereby producing a more natural emotional performance [19]. Although the basic processes are different, the goal of both is usually to show positive emotions. Pugh and James found that these emotions can affect the customer's feelings and the company's results (such as sales, positive recommendations, and repeat customers)[20]. The same situation is found in Douyin's mobile game live streaming.

The discussion above has shown that there is emotional labor in the live streaming of mobile games on Douyin. The live streamer and the audience, as the two main bodies of emotional labor, perform surface acting (the live streamer) and deep acting (the audience). The following is a detailed analysis of the emotional labor of the live streamer and the audience.

\subsection{The Emotional Labor of Mobile Game Live Streamers}

Through emotional labor, live streamers convert their emotions into commercial value and generate income. Moreover, live streaming tends to have low user (audience) stickiness [21]. To achieve superior returns, live streamers need to conceal behaviors that are inconsistent with social norms and values and cater to those values that the public has recognized[22]. Thus, under the combined influence of business logic and the characteristics of live streaming, live streamers must consciously portray themselves in a certain way and feign emotions instead of revealing their true emotions. In this sense, live streamers are carrying out emotional labor by surface acting.

The mobile game live streamers on Douyin focus on the game, and all the contents in the live streaming are developed based on the game. Live streamers need to create a unique emotional space, which is the live room around mobile games to meet the emotional needs of a wider audience. The emotional labor of such live streamers can be summarized as three steps: game selection, game narrative, and interactive activities. The live streamers cultivate fans through these three steps.

The live streamer delineates the basic audience group through game selection. They are already performing emotional labor when choosing which mobile game to stream, as the ultimate goal of game selection is to enable the possibility of higher income by delineating a larger basic audience group. The live streamer's choice of mobile game is therefore often based on the preferences of audiences instead of the live streamer's own preferences. Douyin is a youth-oriented application. To attract larger audiences, therefore, live streamers usually choose mobile games that are popular among Chinese teenagers, such as Glory of Kings and Game for Peace. A popular mobile game can help live streamers quickly attract a large audience and provide scope for audience growth.

In Douyin, view volume is a way to measure the popularity of live streamers. The popularity of live streamers is related to their earnings. Since live streaming has low user (audience) stickiness, after gaining a basic audience group, live streamers need to consolidate this group to ensure that their audience will watch their live streaming. According to Xiao and Hao, the live streamer needs to create a perfect image that meets the expectations of the audience[22]. This situation is the same as the game live streamers on Twitch. Johnson and Woodcock found that live streamers of games on Twitch always strategically show their friendly and humorous side to attract audiences. They call this kind of strategic self-presentation "streaming in character." [23] This strategy can help game live streamers more efficiently manage the pressure of live streaming work and distinguish between their private lives and work. In this case, game live streamers are like actors: What they show in the live streaming is their working image, not the "real" them.

Douyin's mobile game live streamers create their live image through the game narrative. In this way, they can increase the user stickiness and earn greater financial rewards. Using the game narrative, live streamers can form unique image in audiences' minds. Douyin audiences have an exaggerated public impression and image. Their preferred means of expression is also exaggerated[24]. The images of the live streamers therefore have a standard feature, which is exaggeration. In addition, the live streamers can create their live images based on the analysis of their current and potential audiences' gender, age, and preferences.

Although live streamers can attract audiences to watch the live streaming through game selection and game narrative, the audience does not actually have a strong sense of participation. Only when the live streamer and the audience create a fun, intellectual, and interactive social stage will the audience be motivated to watch the whole live streaming for several hours in the live room[25]. Moreover, only when the audience feels the stickiness outside the mobile game in the live room can they become fans of the live streamer. The emotional connection is undeniable when audience members become fans[26]. 
Thus, the live streamer increases the audience's sense of participation in the live streaming by various interactive activities. Moreover, they can also hold a quiz before the game starts to let the audience guess whether they will lose or win. The live streamer can further strengthen the connection to the audience by establishing fan groups. These activities increase the audience's sense of participation in the live streaming, develop the atmosphere in the live room, and give the audience a sense of emotional satisfaction. If the emotional connection between the audience and the live room is strengthened, the fan community will be easier to form. The forming of a fan community indicates the success of the live streamer's fan cultivation. As a result, Douyin's live streamers can also form a more intimate relationship with the audience, ensuring a more stable revenue.

This process of cultivating fans-from nothing to something, from the minority to the majority - can be regarded as the emotional labor of these live streamers. The live streamers mainly rely on a series of emotional operations to attract audiences. From game selection and game commentary to interactive activities, the completion of each instance of emotional labor marks a further step toward fan cultivation. Every emotional operation of the live streamers in their emotional labor receives instant feedback. The live streaming results are visible through data such as traffic, gifts, and the number of fans. The audience is the live streamer's data source. During the fan cultivation process, therefore, the audience dominates the emotional labor of mobile game live streamers on Douyin.

\subsection{The Emotional Labor of the Audience}

Unlike the emotional labor of surface acting, in which true emotion is suppressed and a new emotion is carefully designed by the live streamer, the emotional labor of the audience tends more to deep acting. The emotional labor of the audience is divided into two stages: the emotional labor before becoming a fan and the emotional labor after becoming a fan.

The emotional labor of ordinary audience members is unconscious. They rarely disguise their true emotions. It seems that the ordinary audience members are free to choose their favorite live streaming games, send bullet screens as they like, and send gifts to the live streamer. Their emotions, however, are unconsciously suppressed by the rules of Douyin's live room. The expression of their emotions must be carried out in accordance with the rules; otherwise, they may be muted or blocked. In addition, their acts of watching mobile game live streaming, sending bullet screens, and giving gifts have increased the popularity of live streamers and earned the live streamers income. This result shows that ordinary audience members use the exchange value of emotions. In this study, this kind of emotional labor is considered to be unconscious emotional labor.
After audience members are cultivated by live streamers to become fans, they actively suppress and adjust their true emotions to playing the role of unfettered self-looking for a certain kind of intimacy. In this circumstance, the audience's comments, likes, giftgiving, and other information continue to flow, extend, and diverge into new interactive scenarios[25]. The ultimate goal of fans is to establish an intimate relationship with their favorite live streamers and obtain emotional satisfaction. However, when fans adjust their emotions to establish an intimate relationship with the live streamer, their actions, such as watching the live streaming, sending bullet screens, and giving gifts, all generate income for the live streamer. This shows that fans also use the exchange value of emotions. Whether it is the emotional labor of ordinary audience members or the emotional labor of fans, there are three main manifestations: watching live streaming, sending bullet screens, and giving gifts.

Watching the live streaming is the most basic emotional labor for the audience. Ordinary audience members watch live streaming because of their subjective emotional drive. Their goal is to reach an intellectual and interesting social stage[27]. They can learn game skills from the social stage of live streaming. For fans, there is a situation called "making data." This term is used by Chinese fans to refer to the increase in quantitative data such as reposting, commenting, and reading by fans by bringing attentions to idols in social applications, allowing celebrities to increase their popularity and obtain better career opportunities. In Douyin's live streaming, making data refers to the audience watching the live streaming to maintain the live room's audience numbers and popularity. The purpose of fans who watch mobile game live streaming is to stabilize the live streamer's traffic and ensure the ranking of the live streamer on the Douyin Live Ranking.

Sending bullet screens is another form of emotional labor for the audience. The ordinary audience members and fans can communicate with the live streamer and other audience members in the form of bullet screens. For fans, in addition to communicating and making data, there is another function of the bullet screens: maintaining the reputation of the live streamer. In the relationship between fans and idols, maintaining the reputation of the idols is very important for fans[28]. It is the same for fans of Douyin's mobile game live streamers. If there are rumors about the live streamer, the fans will clarify the situation by sending bullet screens in each live room to maintain the live streamer's reputation. The reputation of the live streamer affects the exchange of the live streamer's emotional labor. In addition, fans send bullet screens out of a need for intimacy. The more bullet screens the fans post, the more likely it is that the audience will be noticed by the live streamer. However, the contents of bullet screens must comply with the rules of the live room. The live streamer or handler of the live 
room may mute or block an audience member who violates the rules. The audience therefore needs to manage their emotions and speak carefully.

Gift-giving is the emotional labor that most reflects the emotional value of the audience and fans. In Douyin, the audience and fans can use virtual currency to buy virtual gifts and reward their favorite live streamers. These virtual gifts are converted into the audience's daily income based on the financial value of the gifts. For ordinary audience members, giving gifts is a way to express their affection. For fans, it is a way to attract the live streamer's attention and give the live streamer financial rewards. In addition, gift-giving can enable fans to upgrade fan cards and deepen the intimate relationship with the live streamer. Fan card is a symbol of fan status. After the audience becomes a fan, the ID of the audience will add the fan logo, which is the fan card. Gift-giving is also the only way for fans to join the fan groups of live streamers. The emotional relationship between the live streamer and the audience and the relationship between audience members are fluid, unstable, and changeable [29]. These relationships are a kind of limited connection. The live streamer's fan group represents the relationship between live streamers and the audience and the relationship between audience members have changed from fluid to relatively stable. This kind of stable relationship is the intimacy that the fans seek. When an audience member who wants to become a fan gets the fan card and joins the fan group of the live streamer, they officially become a fan of the live streamer. This change of identity is the inevitable result of the fan's pursuit of intimacy.

When the audience is converted into fans, the dominant status of fans in the live room changes. Before becoming fans, ordinary audience members have the upper hand in the battle for dominance between live streamers and audiences. The audiences temporarily get rid of the suppression of their real identity and daily life's emotions. After becoming fans, however, they begin to pay attention to the emotional feedback of the live streamer, suppress their emotions, and safeguard the interests of the live streamer. Their emotional labor earns more rewards for the live streamer. The relationship between live streamers and fans can be understood as the relationship between superiors and subordinates in a company. The emotional labor of live streamers is for their own benefit, and the emotional labor of the fans is also for the benefit of live streamers.

However, once the live streamer cannot meet the emotional needs of a fan or deviates from their "perfect image," the fan's attraction to the live streamer may diminish. The fan may choose not to be a fan. The live streamer and the audience therefore restrain each other. The dominant position of the two is constantly changing, and this change in dominance leads to changes in the social interaction. The change in dominance is caused by the emotional labor of live streamers and audiences, and so it can be said that emotional labor has changed the social interaction.

\section{FROM THE VIRTUAL TO REALITY: THE MAGIC CIRCLE TO THE SOCIAL CIRCLE}

Emotional labor triggers new changes in the social structure around Douyin's mobile game live streaming and in the social structure of society. These new changes are brought about by the carnival of Douyin's mobile game live streaming. For Bakhtin, the carnival (the sum of popular celebrations, rituals, and other forms of ceremony) is deeply rooted in the human mind at both the collective and individual levels. Although history is complex, over time, it has formed "a complete language of symbolic concrete perceptual forms," expressing a unified "world carnival sense, permeating all forms"[2]. Douyin can be regarded as a large carnival venue. Live streamers act as performers by showing their game skills and then creating a live image that meets the audience's expectations. Audiences watch live streamers' performances and use bullet screens and gifts to express their feelings. These actions create a free carnival atmosphere in which everyone participates, and everyone achieves a living transcendence of social and personal norms [30]. The mobile game live streaming on Douyin is an example of this kind of carnival.

Bakhtin described how carnivals encourage people to interact and express themselves freely in unity[2]. In the mobile game live streaming on Douyin, this kind of interaction and free expression eventually forms different social circles. For live streamers and audiences, the live room is a carnival arena and a vast magic circle. Based on the original magic circle, live streamers attracted the audience, encouraged their participation, and finally turned the live room into a magic circle where audiences and live streamers interact. Their interaction forms the social relationship between live streamers and audiences and the relationship between members of the audience. This social relationship begins with the remediation of the game by the live streamer.

Remediation is a media theory that focuses on the integration of one kind of media into another. Remediation is a feature of digital media because digital media is constantly remediating its predecessors. Bolter and Grusin proposed the theory of remediation. This theory states that the existence of media is related to other media forms; it is fundamentally comparative and assumes that media does not have autonomous forms or technical characteristics but only exists in other media forms and practices[7]. Murray defines remediation as the phenomenon of copying the conventions or content (or both) of one medium into another medium[31]; for example, adapting a novel into a movie. Mobile game live streaming is also a kind of remediation. It shows the 
content of the game through live streaming. The structure of the live streaming screen allows live streamers and audiences to have the same viewing angle. The live streamer's goal in the mobile game is also the audience's goal, and the live streamer's team is the audience's team. The social relationship between the live streamer and the audience established by the mobile game live streaming is like that of teammates fighting in the same team.

Although the social relationship among members of the audience is also based on the magic circle of games, it is more like a fan community. Through emotional labor, mobile game live streamers on Douyin attract audiences to gather and interact in the world of the magic circle (or live room). In this way, live streamers promote emotional communication and fill the intimacy needs of audiences. According to Utz, society is about building intimate relationships[32]. Similarly, in live streaming, the ultimate goal of the audience's emotional labor is to build intimate relationships. Furthermore, the content of the audience's emotional labor changes with their emotions. From watching the live streaming to sending bullet screens and then to giving gifts, the exchange value contained in the emotional labor gradually increases, meaning that the audience's feelings toward the live room deepen.

The relationship between and status of the audience members in the live room can also be illustrated by the changes in their own emotions. After the audience has been attracted by the emotional labor of the live streamer and converted into fans, the live streamer can establish a fan group and formulate community rules. On Douyin, the emotional labor of fans is identified and classified by the network technology. The intensity of emotion can be measured by specific indicators such as time spent watching, live streaming participation, and gift volume. Otherwise, they run the risk of being rejected from the live streamer's intimate relationship and removed from the fan community.

In addition, the amount of emotional labor that audience members carry out influences their social level in fan community. Any difference in social level only fully emerges after the audience becomes fans. As a unique social network, the fan community conducts group emotional labor with a high degree of organization and institutionalization[33]. Fans usually obey the community rules after entering the fan community so as to gain a sense of belonging, security, and identity within the group. In Douyin's mobile game live streaming, the group emotional labor of individual fans is manifested as a "fan ranking card" or "fan card" for short. The fan card is displayed together with the fan ID. Different fan levels have different visual effects in live rooms. In addition, as the fan level increases, the color of the fan card gradually changes from light to dark. Under this fan hierarchy, fans are divided into management fans and general fans. Management fans are responsible for helping live streamers manage their fan communities and live rooms. The emotional labor of ordinary fans is based on the requirements of the management. The management penalizes fans who violate the community rules. With the power of the community concentrated in the management, management fans and general fans cannot socialize on an equal footing. In contrast, general fans can socialize with one another on a relatively equal footing. Therefore, the social circle of the fan community can be considered a fans' class-based relationship.

The audience in the live room carries out emotional labor because of their need for intimacy. The fan community formed through emotional labor affects the real-life social structure. Bauman uses "liquid" to describe the present era: current society is recognized as a matrix containing all kinds of random connections and separations[34]. This matrix may have countless permutations and combinations. This kind of random connection and separation can be referred to as fluidity. Fluidity is the essence of virtual communities and distinguishes them from traditional communities. The "flow" of the virtual community also includes the flow between the community and society. The virtual community possesses the cohesion and intimacy of a primary group and transcends geographical restrictions. As a virtual community, the hierarchical social structure of the fan community is mapped onto real life through "flow."

In addition, the magic circle of mobile games has continued to expand through live streaming on Douyin and includes audiences in the external, objective world as well as live streamers and mobile games in the internal, virtual world. The audience's group identity, socialization, and the hierarchical system in the external society is affected by the virtual world. The social role of audiences is uncertain, which leads to the blurring and even deconstruction of the boundary between the real society and the virtual world. This deconstruction gives individuals more definitions of social role identities and thus allows them to build more complex social relationships.

Whether it is the "teammate" social relationship between live streamers and audiences or the class-type social circle of the fan community, these new social relationships help to diversify people's social circles. Human society is composed of social networks, and the continuous updating of network technology has given people new methods of socializing. The emergence of mobile game live streaming has further diversified people's methods of socializing and social circle. The social circle is essentially based on the audience's interest in mobile games. The mobile game content is remediated through live streaming, and so introducing various types of real social relationships creates new and virtual social relationships: the teammate social relationship and the fans' class-based relationship. 


\section{CONCLUSION \& DISCUSSION}

The purpose of this paper is to understand the emotional labor in the virtual world through the analysis of the communication among live streamers and audiences of mobile game live streaming on Douyin. The basis of emotional labor is the exchange value of emotion. Through this study, I found a difference in the ultimate purpose of the emotional labor of live streamers and audiences. Live streamers pursue commercial rewards that can sustain their survival, whereas audiences pursue intimate relationships. Live streamers and audiences create a unique social circle through emotional labor. There are two social relationships in this social circle: the "teammate" social relationship between live streamers and audiences, the class-type social circle of the fan community developed by the audiences. A more complex and diversified social interaction has emerged.

Nevertheless, attention must be paid to the chaos brought by emotional labor. The exchange value of emotion leads to the low threshold of the live streaming industry, especially mobile game live streaming. In the premise of not showing the actual image, the threshold of the mobile game live streamer is almost inexistent. This low threshold leads to the uncontrollability of the mobile game live streaming content. The quality of the live streamer's emotional labor cannot be controlled, which leads to the audience being misguided by the value. This carnival of the mobile game live streaming is likely to bring disaster to the whole society. Although there are management regulations like Guiding Opinions on Strengthening the Standardized Management of Live streaming in China, live streaming chaos still exists.

What kind of emotional labor is appropriate? How should emotional labor be regulated? The answers to these questions are not yet known. Emotional labor is a topic worth exploring in depth. This study examines the emotional labor in the virtual world through mobile game live streaming on Douyin. The emotional labor that can be seen elsewhere should not be ignored. I hope that this study can provide some reference for the research on the regulation and rationalization of emotional labor.

\section{REFERENCES}

[1] Bogost, I. (2010). Persuasive games: The expressive power of videogames. Mit Press.

[2] Bakhtin, M. (1984). Problems of Dostoevsky's poetics. U of Minnesota Press.

[3] Huizinga, Johan (1955). Homo Ludens: a Study of the Play-Element in Culture. Boston, MA: The Beacon Press.

[4] Salen, K., Tekinbaş, K. S., \& Zimmerman, E. (2004). Rules of play: Game design fundamentals. MIT press.
[5] Conway, S. (2010). A circular wall? Reformulating the fourth wall for videogames. Journal of Gaming \& Virtual Worlds, 2(2), 145-155.

[6] Wallis, M., \& Shepherd, S. (1998). Studying plays. Bloomsbury Publishing.

[7] Bolter, J. D., Grusin, R., \& Grusin, R. A. (2000). Remediation: Understanding new media. mit Press.

[8] Juul, J. (2001). Games telling stories. Game studies, $1(1), 45$

[9] Chatman, S. (1986). Characters and narrators: Filter, center, slant, and interest-focus. Poetics today, 7(2), 189-204.

[10] Hartmann, T., Wirth, W., Vorderer, P., Klimmt, C., Schramm, H., \& Böcking, S. (2015). Spatial presence theory: State of the art and challenges ahead. Immersed in media, 115-135.

[11] Goleman, D. (1996). Emotional intelligence. Why it can matter more than IQ. Learning, 24(6), 49-50.

[12] Hochschild, A. R. (1979). Emotion work, feeling rules, and social structure. American journal of sociology, 85(3), 551-575.

[13] Cook, A., \& Berger, P. (2000). Predictors of emotion work and household labor among dualearner couples.

[14] Hochschild, A. R. (1990). Ideology and emotion management: A perspective and path for future research. Research agendas in the sociology of emotions, 117, 117-142.

[15] Callahan, J. L., \& McCollum, E. E. (2002). Obscured variability: The distinction between emotion work and emotional labor. Managing emotions in the workplace, 219-231.

[16] Hochschild, A. R. (1983). The Managed Heart: Commercialization of Human Feeling. Berkeley: University of California Press.

[17] Wang, Y. (2019). "New Social Ways": Research on Virtual Interaction of Strangers in Webcast Based on Anchor Perspective. China Youth Study, (2).

[18] Grandey, A. A. (2000). Emotional regulation in the workplace: A new way to conceptualize emotional labor. Journal of occupational health psychology, 5(1), 95.

[19] Grandey, A., Diefendorff, J., \& Rupp, D. E. (Eds.). (2013). Emotional labor in the 21st century: Diverse perspectives on emotion regulation at work (pp.3-17). Routledge.

[20] Pugh, S. D., \& JAMES, M. D. (2013). Emotional 
labor: Organization-level influences, strategies, and outcomes (pp. 219-242). Routledge.

[21] Xun, Y. (2018). Exploration of interactive rituals in live streaming. Academic Exchanges, (5).

[22] Xiao, C., \& Hao, Y. (2018). Performative Behavior in Pan-Entertainment Live Streaming from the Perspective of Mimetic Drama Theory. New Media Research, (12).

[23] Johnson, M. R., \& Woodcock, J. (2019). 'It's like the gold rush': the lives and careers of professional video game streamers on Twitch. tv. Information, Communication \& Society, 22(3), 336-351.

[24] Zhu, Y., \& Yin, W. (2021). Mimetic dramatization marketing in the context of live internet shopping Analysis and interpretation based on Douyin live streamingplatform. Journal Of Shanghai University Of International Business And Economics, (2), 114124.

[25] Yue, L., \& Cai, Q. (2021). Cruising between embedding and de-embedding: the emotional labor of video live streaming fans. Journal Of Fujian Normal University (Philosophy And Social Sciences Edition), (3).

[26] Marwick, A., \& Boyd, D. (2011). To see and be seen: Celebrity practice on Twitter. Convergence, 17(2), 139-158.

[27] Zhu, L., \& Han, Y. (2017). Mimetic Intimacy: A New Look at Foster Idol Fan Communities: A Case Study of TFboys. Contemporary Communication, (6), 76.

[28] Mo, Q. (2009). Popular Culture and Internet Culture (1st ed.). Beijing University of Posts and Telecommunications Press.

[29] Li, Y. (2019). On Gift Giving in Webcasts from the Perspective of Social Network Theory. Social Science Of Beijing, (9), 87-94.

[30] Morson, G. S. (1986). Bakhtin: Essays and dialogues on his work.

[31] Murray, J. H. (2012). Inventing the medium: principles of interaction design as a cultural practice. Mit Press.

[32] Utz, S. (2015). The function of self-disclosure on social network sites: Not only intimate, but also positive and entertaining self-disclosures increase the feeling of connection. Computers in Human Behavior, 45, 1-10.

[33] Hu, C. (2020). From "groupies" to "fandom" --- The "change" and "no change" of fan organizations in China. China Youth Study, (2), 115.
[34] Bauman, Z. (2012). Liquid Times: Living in an Age of Uncertainty. 